\title{
GPD training of dynamic programming-based speech recognizers
}

\author{
Takashi Komori and Shigeru Katagiri \\ ATR Auditory and Visual Perception Research Laboratories, \\ 2-2 Hikaridai, Seika-cho, Soraku-gun, Kyoto, 619-02 Japan
}

(Received 1 February 1992)

\begin{abstract}
Although many pattern classifiers based on artificial neural networks have been vigorously studied, they are still inadequate from a viewpoint of classifying dynamic (variableand unspecified-duration) speech patterns. To cope with this problem, the generalized probabilistic descent method (GPD) has recently been proposed. GPD not only allows one to train a discriminative system to classify dynamic patterns, but also possesses a remarkable advantage, namely guaranteeing the learning optimality (in the sense of a probabilistic descent search). A practical implementation of this theory, however, remains to be evaluated. In this light, we particularly focus on evaluating GPD in designing a widely-used speech recognizer based on dynamic time warping distancemeasurement. We also show that the design algorithm appraised in this paper can be considered a new version of learning vector quantization, which is incorporated with the dynamic programming. Experimental evaluation results in tasks of classifying syllables and phonemes clearly demonstrate GPD's superiority.
\end{abstract}

Keywords: GPD, DTW, LVQ, Neural net, Speech recognition

PACS number: $43.60 . \mathrm{Lq}$

\section{INTRODUCTION}

Applying artificial neural networks (ANNs) to classifier design has attracted considerable interest in the speech recognition field. ${ }^{1-5)}$ In particular, due to the inadequacy that most ANNs such as the multi-layer perceptron are originally suited in terms of structure to handling only a static (fixed-dimensional) vector, developing a satisfactory method of treating a dynamic speech pattern has been one of the most important research topics in this field. In this light, various new network structures have actually been studied: e.g., time delay neural networks $^{1)}$ and a shift-tolerant learning vector quantizer. ${ }^{2)}$ However, compared with the conventional speech recognition approach using the dynamic time warping (DTW) philosophy, the new ANN-motivated approach is not necessarily adequate for representing such dynamics.

An effort to increase the discriminative capability of conventional DTW speech recognizers should thus be a promising alternative to the above ANN approach. These recognizers are usually designed so that class identity, e.g., design sample distribution, can be represented properly. Such a design, without considering classification directly, can not necessarily produce high classification power as a result. Thus, various kinds of improvement have actually been studied in this design framework (e.g. Ref. 6)); however, they unfortunately result in no significant advancement mainly because they all are based on heuristics and not on rigorous theoretical consideration. Motivated by this concern, one of the authors and his colleagues proposed a new discriminative learning theory, namely GPD, as one solution to this current difficulty. ${ }^{7,8)}$ GPD is a novel learning framework formalized by generalizing the classical probabilistic descent method which was developed a quarter of a century ago, ${ }^{9)}$ and includes a family of new discriminative training 
ideas for various kinds of classifier structures. However, due to its intrinsic generality and complexity of formulation, careful evaluation of implementing GPD still remains an emerging research question, though very recently several evaluation studies have actually been started. ${ }^{10-12)}$ This paper is intended to show the results of our research which is a continuation of this evaluation.

There are two main DTW approaches: one is based on a DP-based distance classifier (DPC) and one is based on a hidden Markov model (HMM). Comparing DPC and HMM, distance calculation which is time-consuming, but indispensable in DPC, is often criticized. Such criticism, however, holds true only in the scheme of traditional sequential computation. Remarkable progress in recent parallel computation and hardware technology, such as the advent of a fine-grained parallel machine, would remind one of the usefulness of a pattern classifier consisting of simple distance calculation (e.g., Ref. 13)). Thus, in this paper, we selected DPC, which has long been studied in speech recognition, as our implementation framework.

One of the main goals in this paper is to evaluate a GPD-based discriminative training algorithm in designing a multi-reference DPC. We first introduce a somewhat complicated but general training rule based on the full knowledge of GPD; this rule is referred to as the G-rule. Detailed evaluation of the G-rule will be presented separately. ${ }^{12)}$ However, to increase awareness on application possibilities, we particularly focus on evaluating a more practical and simpler algorithm which can be defined as an extremely simple case of the G-rule; this simple algorithm is referred to as the S-rule. Experimental results using this S-rule actually demonstrate that GPD greatly contributes to increasing classification accuracy.

Interestingly, it is also shown that the S-rule can be viewed as an idea of learning vector quantization (LVQ) generalized so as to be suited to the use of DP. An alternative to such integration of LVQ and DP would occur to anyone recalling several intuitive hybrid ideas developed by combining existing algorithms (e.g., Refs. 6, 14, 15)). However, without doubt, the theoretical appropriateness of a new algorithm should be proved. It is here worthwhile noting that GPD-based rules are guaranteed to be optimal from the viewpoint of a probabilistic descent search because each of them fundamentally inherits all properties of GPD.

This paper is organized as follows. Section 2 will be used to introduce the G-rule in detail. In Section 3, we will first propose the S-rule by simplifying the G-rule and next show that the S-rule can be treated as a generalized LVQ to categorize dynamic patterns. In Section 4, we will show the experimental results on two tasks: English isolated syllable classification and classification of Japanese phonemes extracted from word utterances. The paper will be concluded in Section 5 .

\section{GPD TRAINING FOR MULTI- REFERENCE DPC; G-RULE}

Two ideas, DTW and DP, are often used to represent the same technique in speech recognition. However, for clarity we will define these two ideas before starting our main discussion. In this paper, we define DTW as a general concept of nonlinear time warping which allows one to treat any possible, reasonable, time-alignment path between an input and a reference; on the other hand, we define DP as one specific class of DTW-based algorithms, which is usually pursued by the dynamic programming best-path search.

We here present the G-rule by accurately following the GPD formalization for a multi-reference DPC. ${ }^{7,8,10-12)}$ Consider an $M$-class task $C_{m}(m=$ $1,2, \ldots, M)$. We assume that a speech utterance $\boldsymbol{x}$ is a variable but finite length sequence of acoustic feature vectors. Each acoustic feature vector has a fixed dimension $(S)$, usually consisting of coefficients based on the linear predictive coding or the Fourier transform. $\boldsymbol{x}_{t}$ denotes the $t$-th acoustic feature vector (frame) of $\boldsymbol{x}$. We also assume that a classifier consists of a set of reference vectors

$$
\begin{aligned}
\Lambda= & \left\{\lambda_{m}=\left\{\boldsymbol{r}_{m}{ }^{b}\right\}, b=1,2, \ldots, B_{m},\right. \\
& \text { and } m=1,2, \ldots, M\},
\end{aligned}
$$

where $\boldsymbol{r}_{m}{ }^{b}$ denotes the $C_{m}$ 's reference pattern $b$-th closest to $\boldsymbol{x}$, and $B_{m}$ is the number of reference patterns for $C_{m} . \quad \boldsymbol{r}_{m}{ }^{b}$ is also a variable but finite duration $(T)$ sequence of acoustic feature vectors.

We consider a sequential, or adaptive, training scheme where a classifier is adjusted by a small amount every time a single training token is given. According to the GPD idea, we introduce the following measurable distances. First, to measure the degree to which $\boldsymbol{x}$ belongs to $C_{m}$, a discriminant function, or $a$ class distance, is defined by 


\section{T. KOMORI and S. KATAGIRI: GPD TRAINING OF DP-BASED SPEECH RECOGNIZERS}

$$
g_{m}(\boldsymbol{x} ; \Lambda)=\left[\sum_{b=1}^{B m}\left\{D\left(\boldsymbol{x}, \boldsymbol{r}_{m}^{b}\right)\right\}^{-\zeta}\right]^{-1 / \zeta},
$$

where $D\left(\boldsymbol{x}, \boldsymbol{r}_{m}{ }^{b}\right)$ is called a reference distance between $\boldsymbol{x}$ and $\boldsymbol{r}_{m}{ }^{b}$, and $\zeta$ is a positive number. The reference distance is also defined in the same fashion as (2);

$$
D\left(\boldsymbol{x}, \boldsymbol{r}_{m}^{b}\right)=\left[\sum_{\theta=1}^{\theta}\left\{D_{\theta}\left(\boldsymbol{x}, \boldsymbol{r}_{m}^{b}\right)\right\}^{-\xi}\right]^{-1 / \hat{\xi}},
$$

where $D_{b}\left(\boldsymbol{x}, \boldsymbol{r}_{m}{ }^{b}\right)$ is a path distance accumulated along the $\theta$-th best (smallest distance) path selected by the DP-matching between $x$ and $\boldsymbol{r}_{m}{ }^{b}$ among all the possible $\Theta$ paths. Here, the path distance is decomposed as

$$
D_{\theta}\left(x, \boldsymbol{r}_{m}{ }^{b}\right)=\sum_{t=1}^{T} w_{m, t}^{b} \delta_{m, \theta_{t}}^{b},
$$

where $w_{m, t}^{b}$ is a weighting factor corresponding to the $t$-th frame of $\boldsymbol{r}_{m}{ }^{b}, \delta_{m, \theta_{t}}^{b}$ is a local distance between the $t$-th frame of $\boldsymbol{r}_{m}{ }^{b}$ and the corresponding frame $\theta_{t}$ of $\boldsymbol{x}$ along the $\theta$-th best path. For simplicity, in this paper we treat the weighting factor as a nontrainable constant, though it was trained in Refs. $10,12)$. There are many possible ways of measuring the local distance, but by way of example we use here the Euclidean distance

$$
\delta_{m, \theta_{t}}^{b}=\sum_{s=1}^{S}\left(\boldsymbol{r}_{m, t, s}^{3}-\boldsymbol{x}_{\theta_{t}^{m, b}, s}\right)^{2},
$$

where $\boldsymbol{r}_{m, t, s}^{b}$ and $\boldsymbol{x}_{\theta_{t}^{m, b, s}}$ are the $s$-th elements of $\boldsymbol{r}_{m, t}^{b}$ and $\boldsymbol{x}_{\theta_{t}^{m, b}}$ respectively, and $\boldsymbol{x}_{\theta_{t}^{m, b}}$ is the frame of $\boldsymbol{x}$ corresponding to $\boldsymbol{r}_{m, t}^{b}$ along the $\theta$-th best path.

There are again many possible decision (classification) rules, each using the class distances, and we here simply choose the following rule

$$
C(x)=C_{i}, \quad \text { if } i=\underset{j}{\operatorname{argmin}}\left\{g_{j}(x ; \Lambda)\right\} .
$$

Our target is to train $\Lambda$ such that misclassifications can be minimized given this decision rule. To perform this training, classification results should be embedded in a functional form which some reasonable optimization method, such as the gradient search, can treat properly. In fact, GPD demonstrated that there were many possible ways for this embedding to take place. In particular, we here use the misclassification measure

$$
d_{k}(\boldsymbol{x})=g_{k}(\boldsymbol{x} ; \Lambda)-\left[\frac{1}{M-1} \sum_{j, j \neq k}\left\{g_{j}(\boldsymbol{x} ; \Lambda)\right\}^{-1 / \mu}\right]
$$

where $\mu$ is a positive number, for a given training token $\boldsymbol{x} \in C_{k}$. One notes that a larger $d_{k}(\boldsymbol{x})$ implies a more definite misclassification of $\boldsymbol{x}$ and a negative $d_{k}(x)$ implies a correct classification.
A general form of cost function is next given as

$$
l_{k}(\boldsymbol{x} ; \Lambda)=l_{k}\left(d_{k}(\boldsymbol{x} ; \Lambda)\right),
$$

where $l_{k}$ is a monotonically increasing, differentiable function. It is worthwhile noting that the selection of $l_{k}$ leads to various implementations of training criteria, but the following function

$$
l_{k}\left(d_{k}(x ; \Lambda)\right)=\frac{1}{1+e^{-\alpha\left(d_{k}(x ; \Lambda)+\beta\right)}}, \quad \alpha>0
$$

properly approximates the most important classification criterion, namely, the minimum classification error criterion (See Ref. 16)). In this light, we will use Eq. (9) in the discussion below.

GPD guarantees that adjusting $\Lambda$ by

$$
-\epsilon \boldsymbol{U} \nabla l_{k}(\boldsymbol{x} ; \Lambda),
$$

where $\epsilon$ is a small positive number and $U$ is a positive definite matrix, leads to at least a locally optimal minimum error classifier in a probabilistic sense; this convergence property was the main result of the original probabilistic descent method. ${ }^{9)}$ An actual adjustment rule in our situation is given by

$$
\begin{array}{r}
\boldsymbol{r}_{m, t, s}^{b}(n+1)=\boldsymbol{r}_{m, t, s}^{b}(n)-2 \epsilon_{n} \nu_{k} w_{m, t}^{b} \phi_{m} \psi_{m} \tau_{m}, \\
\text { for } m=k, \\
\boldsymbol{r}_{m, t, s}^{b}(n+1)=\boldsymbol{r}_{m, t, s}^{b}(n)+2 \frac{\epsilon_{n} \nu_{k} w_{m, t}^{b}}{M-1} \kappa_{m} \phi_{m} \psi_{m} \tau_{m}, \\
\text { for } m \neq k,
\end{array}
$$

where

$$
\begin{aligned}
& v_{k}=l_{k}{ }^{\prime}\left(d_{k}(\boldsymbol{x} ; \Lambda)\right)=\alpha l_{k}\left(d_{k}(\boldsymbol{x} ; \Lambda)\right)\left\{1-l_{k}\left(d_{k}(\boldsymbol{x} ; \Lambda)\right)\right\}, \\
& \phi_{m}=\left[\sum_{b^{\prime}=1}^{B m}\left\{\frac{D\left(\boldsymbol{x}, \boldsymbol{r}_{m}{ }^{b}\right)}{D\left(\boldsymbol{x}, \boldsymbol{r}_{m}{ }^{\prime}\right)}\right\}^{\zeta}\right]^{-(1+\zeta) / \zeta}, \\
& \psi_{m}=\left[\sum_{\theta=1}^{\theta}\left\{D_{\theta}\left(\boldsymbol{x}, \boldsymbol{r}_{m}^{b}\right)\right\}^{-\xi}\right]^{-(1+\xi) / \xi} \text {, } \\
& \tau_{m}=\left[\sum_{\theta=1}^{\theta} \frac{\left(\boldsymbol{r}_{m, t, s}^{b}-\boldsymbol{x}_{t}^{m, b, s}\right)}{\left\{D_{\theta}\left(\boldsymbol{x}, \boldsymbol{r}_{m}^{b}\right)\right\}^{\xi+1}}\right], \quad \text { and } \\
& \kappa_{m}=\left[\frac{1}{M-1} \sum_{j, j \neq k}\left\{\frac{g_{m}(x ; \Lambda)}{g_{j}(x ; \Lambda)}\right\}^{\mu}\right]^{-(1+\mu) / \mu} \text {. }
\end{aligned}
$$

Equation (10) gives the complete G-rule procedure. Although we only consider handling dynamic patterns in this paper, our result is also applicable to static vector classification because a static vector is merely a limited case of a dynamic pattern.

\section{SIMPLIFIED TRAINING; S-RULE}

Equation (10) illustrates that all the reference vectors will be adjusted along all the time warping paths every time a single training token is given. However, the computation of all these possible cases 
would be very time-consuming, and a reasonable way of simplifying Eq. (10) should be attempted.

There are obviously various possibilities in implementing Eq. (10), due to the selection of the parameters such as $\mu$. In this section, we particularly consider an extremely simplified case of G-rule, by setting $\mu, \zeta, \xi \rightarrow \infty$. This kind of extreme simplification was discussed in Ref. 7) for the case of classifying static vectors and, in fact, a crucial link between LVQ and GPD was revealed. The extreme setting results in the following manifolds.

$$
d_{k}(\boldsymbol{x}) \approx g_{k}(\boldsymbol{x} ; \Lambda)-g_{i}(\boldsymbol{x} ; \Lambda),
$$

where $C_{i}(i \neq k)$ is the most probable among the incorrect classes.

$$
\begin{aligned}
& g_{m}(\boldsymbol{x} ; \Lambda) \approx D\left(\boldsymbol{x}, \boldsymbol{r}_{m}{ }^{1}\right) \\
& D\left(\boldsymbol{x}, \boldsymbol{r}_{m}{ }^{1}\right) \approx D_{1}\left(\boldsymbol{x}, \boldsymbol{r}_{m}{ }^{1}\right)
\end{aligned}
$$

Here, one should note the following three points: 1) due to Eq. (11), only two classes, the correct class and the most probable among the incorrect classes, concern the classification decision; 2) each class distance is represented by only the reference closest to the input among the same class references; 3 ) the reference distance is measured along only the corresponding best path usually selected using the DPbased minimum search operation. Equation (10) is then reduced into

$$
\begin{array}{r}
\boldsymbol{r}_{m, t, s}^{b}(n+1)=\boldsymbol{r}_{m, t, s}^{b}(n)-2 \epsilon_{n} \nu_{k} \boldsymbol{w}_{m, t}^{b}\left(\boldsymbol{r}_{m, t, s}^{b}(n)-\boldsymbol{x}_{1}^{m, b, s}\right), \\
\text { for } m=k \text { and } b=1, \\
\boldsymbol{r}_{m, t, s}^{b}(n+1)=\boldsymbol{r}_{m, t, s}^{b}(n)+2 \epsilon_{n} \nu_{k} w_{m, t}^{b}\left(\boldsymbol{r}_{m, t, s}^{b}(n)-\boldsymbol{x}_{1 t}^{m, b}, s\right), \\
\quad \text { for } m=i \text { and } b=1, \\
\boldsymbol{r}_{m, t, s}^{b}(n+1)=\boldsymbol{r}_{m, t, s}^{b}(n), \quad \text { otherwise , }
\end{array}
$$

where $n$ is a discrete time index in training, $\epsilon_{n}$ is a small positive number, and $1_{t}^{k, 1}=\left.\theta_{t}^{k, 1}\right|_{\theta=1}$.

The optimality of probabilistic descent search requires $\epsilon_{n}$ to meet the conditions in Ref. 9), originally based on the stochastic approximation philosophy. However, these conditions (assuming infinite training repetition) are actually never realistic. Thus, we reasonably approximate $\epsilon_{n}$ as

$$
\epsilon_{n}=\epsilon_{0}\left(1-\frac{n}{N}\right),
$$

where $\epsilon_{0}$ is a positive small number and $N$ is a large prescribed positive constant.

We next consider the simplification of the deriva- tive form of the loss $v_{k}$. The form $v_{k}$ is originally a symmetric function of $d_{k}(\boldsymbol{x})$, i.e. unimodal around an actual class boundary. See Fig. (1). Taking account of the fact that the learning-when-incorrect is essential for minimizing misclassifications on training data, we replace $v_{k}$ by the following step function

$$
\omega=\left\{\begin{array}{lc}
1, & \rho_{1}<d_{k}(x)<\rho_{2}, \\
0, & \text { otherwise },
\end{array}\right.
$$

where $\rho_{1}$ is zero and $\rho_{2}$ is a positive constant, or

$$
\omega=\left\{\begin{array}{lc}
1, & \rho_{1}^{\prime}<\frac{d_{k}(\boldsymbol{x})}{d_{i}(\boldsymbol{x})}<\rho_{2}^{\prime}, \\
0, & \text { otherwise },
\end{array}\right.
$$

where $\rho_{1}^{\prime}$ is positive constant less than 1 and $\rho_{2}{ }^{\prime}$ is 1 . One may note that $\omega$ formally corresponds to the derivative of a piece-wise linear step function approximating the smooth sigmoid loss (See Fig. 2). Equation (14) is now revised to

$$
\begin{array}{r}
\boldsymbol{r}_{m, t, s}^{b}(n+1)=\boldsymbol{r}_{m, t, s}^{3}(n)-2 \epsilon_{n}\left(\omega w_{m, t}^{b}\left(\boldsymbol{r}_{m, t, s}^{b}(n)-\boldsymbol{x}_{t}^{m, b, s}\right),\right. \\
\text { for } m=k \text { and } b=1, \\
\boldsymbol{r}_{m, t, s}^{b}(n+1)=\boldsymbol{r}_{m, t, s}^{b}(n)+2 \epsilon_{n} \omega w_{m, t}^{b}\left(\boldsymbol{r}_{m, t, s}^{b}(n)-\boldsymbol{x}_{t}^{m, b, s}\right), \\
\quad \text { for } m=i \text { and } b=1, \\
\boldsymbol{r}_{m, t, s}^{b}(n+1)=\boldsymbol{r}_{m, t, s}^{b}(n), \quad \text { otherwise },
\end{array}
$$

We define here the rule in Eq. (17) as the S-rule. The resultant training procedure using the S-rule is summarized as follows:

1. For each training token, $\boldsymbol{x}(n), n=1,2, \ldots, N$, do steps $2-7$. sequentially.

2. Compute the misclassification measure, $d_{k}(\boldsymbol{x})$.

3. For each class, $C_{m}, m=1,2, \ldots, M$, do steps $4-7$.

4. For each reference in $C_{m}, \boldsymbol{r}_{m}{ }^{b}, b=1,2, \ldots, B_{m}$, do steps 5-7.

5. For each frame in $\mathbf{r}_{m}{ }^{b}, \mathbf{r}_{m, t}^{b}$, do steps 6-7.

6. For each element in $\boldsymbol{r}_{m, t}^{b}, \boldsymbol{r}_{m, t, s}^{b}$, do step 7 .

7. Adjust the elements, $\boldsymbol{r}_{m, t, s}^{b}$, according to Eq. (17).

The above discussion would already suggest that the LVQ adjustment philosophy underlies the Srule. We discuss the S-rule in the view of a generalized LVQ in the remainder of this section. Consider the case in Fig. 1 (a). Equation (17) then implies that the adjustment occurs only for the misclassification that incurred around the actual class boundary. It is now obvious that the function $\omega$ substantially works as the vector space window of 


\section{T. KOMORI and S. KATAGIRI: GPD TRAINING OF DP-BASED SPEECH RECOGNIZERS}

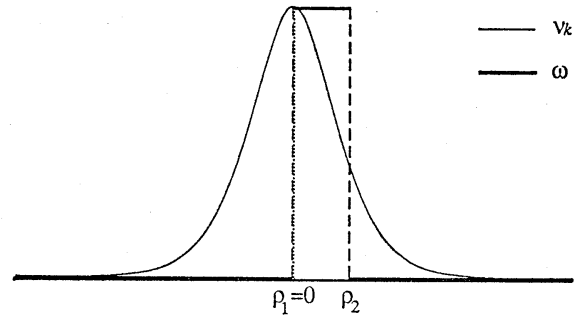

(a)

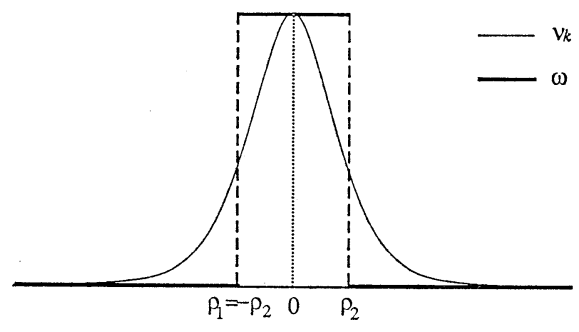

(b)

Fig. 1 Derivative form of loss $\left(\nu_{k}\right)$ and its approximation $(\omega)$. The form $\omega$ in (a) is designed to minimize misclassifications over training data, and the form $\omega$ in (b) is expected to create a robust class boundary for unknown data. The ordinate is here normalized.

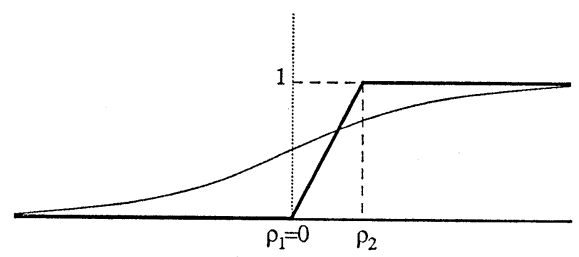

Fig. 2 The form of loss (thin line) and a sample of its approximation (thick line).

LVQ. ${ }^{17)}$ We then call the function $\omega$ a space window hereafter. One should note here that the adjustment strategy of Eq. (17) at each frame position is equivalent to the modified version of LVQ2. ${ }^{18)}$ Our simplification eventually leads to the following; an extremely simple GPD training rule for DPC, namely the $S$-rule, is equivalent to the generalized idea of performing the LVQ training along the DP best path between a dynamic input and the closest reference pattern.

\section{EXPERIMENTS}

Experiments were conducted on two sets of isolated speech tokens: 1) English E-rhyme letters (E-set), and 2) Japanese phonemes (P-set).

\section{$4.1 \quad$ E-set}

The E-set is the task of classifying nine English E-rhyme letters, i.e. $\{\mathrm{b}, \mathrm{c}, \mathrm{d}, \mathrm{e}, \mathrm{g}, \mathrm{p}, \mathrm{t}, \mathrm{v}, \mathrm{z}\}$. Speech tokens were recorded over telephone lines by one hundred untrained speakers: 50 female and 50 male speakers, which were then converted to acoustic feature vectors using the conditions shown in Table 1. Each speaker voiced each E-rhyme letter twice, once for training and once for testing. Due to their high confusability because of the common following sound $/ \mathrm{i}:$, this task has long been used as a good framework to evaluate many different classifiers. Actually it was reported that one of the baseline systems, namely, a system consisting of continuous HMMs (5-component mixture Gaussian distributions, 5-state left-to-right structure, and no skip) produced only $61.7 \%$ on unknown testing data $\left(80.2 \%\right.$ on training data). ${ }^{14)}$

There are many possible ways selecting the system size, or the number of references. By way of example, we used the following two classifiers: one consisting of only one reference pattern per class $(R l)$ and one consisting of three reference patterns per class $(R 3)$. Each classifier was first initialized using the modified $k$-means clustering method; two versions of this clustering idea, the minimax method and the pseudo-average method, were adopted for the sake of reliable comparison. ${ }^{19)}$ We then ran the S-rule training for each classifier. Taking account of the finiteness of available training tokens, we repeated full presentation of the training data with shuffling each time. We refer to this full presentation as epoch. We ran 20 epochs through the experiments.

Table 1 Acoustic feature extraction conditions for E-set.

\begin{tabular}{ll}
\hline Sampling frequency & $6.67 \mathrm{kHz}$ \\
\hline Time window & 45 -ms Hamming, 15-ms shift \\
\hline Acoustic feature vectors & $\begin{array}{l}\text { 24-dimension } \\
\text { (12-dim LPC cepstrum \& } \\
\text { 12-dim delta cepstrum) }\end{array}$ \\
\hline
\end{tabular}


Figure 3 shows a typical training curve, i.e., a recognition rate over training data $v s$. an epoch. This curve demonstrates that the S-rule can achieve a very high discriminative power, quickly and steadily.

Table 2 lists the results for several different conditions. We here used the space window Eq. (16b). The conditions were selected according to our preliminary experiments. In particular, the following parameters were fixed as follows: $N=18,000$ ( $=9$ letters $\times 100$ speakers $\times 20$ epochs), $\epsilon_{0}=0.02$, and $w_{m, t}^{b}=1$. Since the selection of $\rho_{1}^{\prime}$ seems to be closely related to the focus on class boundary estimation, we compared several choices for $\rho_{1}{ }^{\prime}$. The classifier R1 was trained with $\rho_{1}{ }^{\prime}=0.9$, while the classifier R3 was trained with $\rho_{1}{ }^{\prime}=0.5,0.6,0.7,0.8$, 0.9. All results for R3 were similar and only the best results, one for each selection of $\rho_{1}{ }^{\prime}$, and listed in the table $\left(\rho_{1}{ }^{\prime}=0.5\right.$ for the minimax clustering,

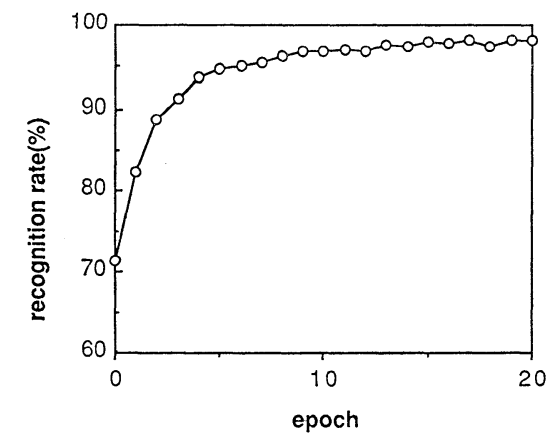

Fig. 3 A typical training curve on the Eset task. The recognition rate on training data rises quickly and smoothly.

Table 2 Recognition rates in the E-set task.

\begin{tabular}{|c|c|c|c|c|}
\hline $\begin{array}{l}\text { Initial reference } \\
\text { clustering } \\
\text { method }\end{array}$ & $\begin{array}{l}\text { Number } \\
\text { of refs. }\end{array}$ & $\begin{array}{c}\text { Before/ } \\
\text { after } \\
\text { S-rule } \\
\text { training }\end{array}$ & $\begin{array}{l}\text { Results } \\
\text { training }\end{array}$ & $\begin{array}{l}\text { Results } \\
\text { testing }\end{array}$ \\
\hline Minimax & R1 & before & $56.7 \%$ & $55.0 \%$ \\
\hline Minimax & R1 & after & $99.0 \%$ & $74.2 \%$ \\
\hline Pseudo-avg. & R1 & before & $61.3 \%$ & $59.8 \%$ \\
\hline Pseudo-avg. & R1 & after & $98.9 \%$ & $74.9 \%$ \\
\hline Minimax & R3 & before & $72.4 \%$ & $64.1 \%$ \\
\hline Minimax & R3 & after & $100.0 \%$ & $72.4 \%$ \\
\hline Pseudo-avg. & R3 & before & $71.3 \%$ & $64.9 \%$ \\
\hline Pseudo-avg. & R3 & after & $99.8 \%$ & $74.0 \%$ \\
\hline
\end{tabular}

and $\rho_{1}{ }^{\prime}=0.6$ for the pseudo-avg. clustering). Regardless of the selection of the $k$-means clustering procedures, the difference in classification power between the initial situation and the after-training situation is obvious. Without doubt, the results illustrate the high superiority of our GPD-based training. In particular, the training achieved a significant reduction in recognition rates over the training data, ranging from $30 \%$ to $45 \%$, as well as an almost perfect accuracy over the training data.

As we pointed out, the adjustment only for misclassification cases is enough to reduce misclassifications over the design samples. However, due to the finite number of design samples, the training even for correct classification cases would help increase the accuracy for future unknown data. ${ }^{20-21)}$ We then treat another space windows; assuming $\rho_{1}=-\rho_{2}$ for Eq. (16a) (See Fig. 1 (b)), and $\rho_{2}{ }^{\prime}=1 / \rho_{1}{ }^{\prime}$ for Eq. (16b).

This implies that the adjustment could occur even if a training token is correctly but somehow insecurely classified. Although further analysis is certainly required, it would be expected that this symmetric window creates a robust class boundary for unknown data (e.g., Ref. 16)). Table 3 shows results for this new space window. Here, the same conditions with those in the above paragraph were used. As above, the rate listed for R3 is the best result among the five different $\rho_{1}{ }^{\prime \prime}$ s $\left(\rho_{1}{ }^{\prime}=0.7\right.$ for minimax clustering, and $\rho_{1}^{\prime}=0.8$ for pseudo-avg. clustering). The results again show the superiority of the S-rule and also suggest the plausibility of the above discussion as concerns robustness.

In parallel with our study, several high recognition rates on this E-set have also been reported in succession..$^{8,10,12,14)}$ It should be pointed out here that GPD underlies each of these promising approaches.

Table 3 Recognition rates on the E-set task using a symmetric space window.

\begin{tabular}{lcccc}
\hline $\begin{array}{c}\text { Initial } \\
\text { reference } \\
\text { clustering } \\
\text { method }\end{array}$ & $\begin{array}{c}\text { Number } \\
\text { of refs. }\end{array}$ & $\begin{array}{c}\text { Before/ } \\
\text { after } \\
\text { S-rule } \\
\text { training }\end{array}$ & $\begin{array}{c}\text { Results } \\
\text { training }\end{array}$ & $\begin{array}{c}\text { Results } \\
\text { testing }\end{array}$ \\
\hline minimax & R1 & after & $94.1 \%$ & $75.4 \%$ \\
pseudo-avg. & R1 & after & $96.1 \%$ & $74.9 \%$ \\
minimax & R3 & after & $96.8 \%$ & $77.2 \%$ \\
pseudo-avg. & R3 & after & $98.1 \%$ & $76.7 \%$ \\
\hline
\end{tabular}




\section{T. KOMORI and S. KATAGIRI: GPD TRAINING OF DP-BASED SPEECH RECOGNIZERS}

4.2 P-set

Although the above results have not reset the record on the E-set (e.g., see Ref. 8)), the improvement in recognition rates clearly demonstrated the effectiveness of the proposed algorithm. However, the E-set consisting of only the E-rhyme letters is very special from the viewpoint of speech recognition taks. We thus tested the algorithm on the second data set consisting of the possible Japanese phonemes, namely the P-set.

The P-set is a set of phoneme segments extracted from the ATR 5,240 Japanese common word data, using manually-selected acoustic-phonetic labels. ${ }^{22)}$ This set was split into two independent sets of roughly equal sizes: one for training and one for testing. Each word was spoken in a soundproof booth by one male professional announcer and was transformed to a sequence of 16-dimensional Mel-scale spectrum vectors using the parameters specified in Table 4. We then used a 112-dimensional acoustic feature vector, concatenating 7 adjacent Mel-scale spectrum vectors. This high-dimensional vector was also normalized so that the average of all the vector components was 0.0 .

To classifier was composed of 5 reference patterns per class, for 53 classes. Each of the background

Table 4 Acoustic feature extraction conditions for P-sets.

\begin{tabular}{ll}
\hline Sampling frequency & $12 \mathrm{kHz}$ \\
\hline Time window & 20-ms Hamming, 5-ms shift \\
\hline Acoustic feature vectors & $\begin{array}{c}\text { 16-dimension Mel-scale } \\
\text { coefficients } \\
\text { (down-sampled from 256- } \\
\text { point FFT coefficients) }\end{array}$ \\
\hline
\end{tabular}

segments and contracted sounds such as $/ \mathrm{kj} /$ was treated as one class. A word-initial vowel was categorized separately from a class of inside vowels and word-final vowels: e.g., a phoneme token /a/ in the word beginning was treated as a different class token compared to a token /a/ from the other segment positions. Moreover, a long vowel such as /o:/ in the word /o:ki:/ was categorized as different from a typical vowel class. It should, however, be noticed that, in the recognition phase, the classifier did not distinguish the vowel position, either wordinitial or other; this implies that classification was evaluated for only 41 classes. Table 5 lists the 41 phoneme categories used in recognition.

Taking account of the experimental results in the previous section, here we used the minimax method and the robust version of space window $\left(\rho_{2}{ }^{\prime}=1 / \rho_{1}{ }^{\prime}\right)$. We performed 10 epochs of training on 13,626 training tokens $(N=136,260)$, with $\epsilon_{0}=0.05, \rho_{1}{ }^{\prime}=0.8$, and $w_{m, t}^{b}=1$. We achieved $98.4 \%$ over training data and $96.2 \%$ over testing data. This result shows that our discriminative training algorithm works well not only on small, special tasks such as E-sets, but also on ordinary tasks with a large number of classes. Although recognition rates by other algorithms have not been reported for the same class arrangement, namely 41 classes, our results can be considered rather high. Compare them with $95.3 \%$ by the shift-tolerant LVQ and $97.2 \%$ by LVQHMM, both of which were obtained in a 25 -class condition using extractly the same database. ${ }^{6)}$ Consequently, the results here again demonstrated the very high discriminative power of the S-rule.

\section{CONCLUSION}

We presented the new GPD-based discriminative

Table 5 Forty one phoneme categories of the P-set.

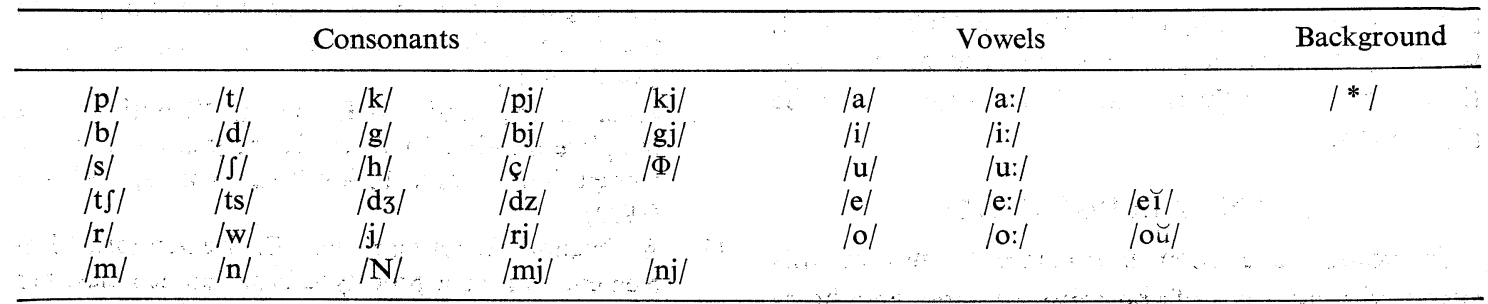

Note 1: Each of $/ \mathrm{h} /, / \mathrm{c} /$ and $/ \Phi /$ denotes the initial phoneme of the Japanese syllable $\{$ ha, hi, he, ho\}, \{hya, hyu, hyo $\}$ and $\{$ hu $\}$ respectively.

Note $2: / \mathbf{N} /$ includes $/ \mathrm{m} /, / \mathrm{n} /$ and $/ \mathrm{j} /$, each corresponding to the Japanese kana $ん$.

Note $3: / * /$ denotes a background segment. 
training algorithms, the G-rule and the S-rule, for a multi-reference distance classifier handling dynamic patterns. We also showed that the simpler S-rule could be viewed as a generalized LVQ for dynamic pattern classification. In other words, the S-rule is actually equivalent to a hybrid of the LVQ rule and the DP-based best path search. However, it should be stressed here that the DP search is not essential to the optimality pursued in our approach; the DPsearch is merely used for simplification and its optimality in minimum operation is not crucial in our optimization based on the gradient search. This point is quite different from several algorithms where the DP-based optimality is used as a part of training optimality (e.g., see Ref. 3)).

The simple and practical S-rule was evaluated in the two isolated-mode speech recognition tasks. The experimental results clearly demonstrated that the proposed algorithm contributed towards increasing the discriminative power of the traditional DP-based speech recognizer.

The experiments reported here were designed to show the fundamental effectiveness of our novel algorithms. The superiority of the S-rule was clearly demonstrated. However, the characteristics of our algorithms, particularly of the G-rule, must be studied further. Specifically, using finite values of classification parameters such as $\xi$ in the G-rule would contribute toward increasing classifier robustness, which is an emerging ANN research topic, because the G-rule intrinsically possesses the promising property of smooth decision making based on multi-reference, multi-path, and multi-class measurement. ${ }^{12)}$ The proposed algorithms should also be studied in a more realistic task such as connected speech recognition.

It is lastly worthwhile noting that, without any serious modification, the main formulations in this paper are applicable to the so-called batch-type gradient search, e.g., the steepest descent method, where all the training tokens are used at the same time for every adjustment. This point would be also tested.

\section{ACKNOWLEDGEMENTS}

It would have been impossible to pursue this study without the collaboration of, and enlightening discussions with, Dr. Biing-Hwang Juang and Dr. Chin-Hui Lee. The study was supported by many people at AT\&T Bell Laboratories and ATR
Auditory and Visual Perception Research Laboratories, particularly Dr. Lawrence Rabiner and Dr. Yoh'ichi Tohkura. Mr. Hitoshi Iwamida guided us in beginning the experiments. The authors would like to deeply thank all of them for their great help.

\section{REFERENCES}

1) A. Waibel, T. Hanazawa, G. Hinton, K. Shikano, and $\mathrm{K}$. Lang, "Phoneme recognition: Neural networks vs. hidden Markov models," Proc. ICASSP88, Vol. 1, 107-110 (1988).

2) E. McDermott and S. Katagiri, "Shift-invariant, multi-category phoneme recognition using Kohonen's LVQ2," Proc. ICASSP-89, Vol. 1, 8184 (1989).

3) K. Iso and T. Watanabe, "Speaker-independent word recognition using a neural prediction model," ICASSP-90, Vol. 1, 441-444 (1990).

4) E. Levin, "Word recognition using hidden control neural architecture," Proc. ICASSP-90, Vol. 1, 433436 (1990).

5) T. Kohonen, "Learning vector quantization," Helsinki Univ. of Technology, Report TKK-F-A601 (1986).

6) H. Iwamida, S. Katagiri, and E. McDermott, "A hybrid speech recognition system using HMMs with an LVQ-trained codebook," J. Acoust. Soc. Jpn. (E) 11, 277-286 (1990).

7) S. Katagiri, C.-H. Lee, and B.-H. Juang, "A generalized probabilistic descent method," Proc. Autumn Meet. Acoust. Soc. Jpn. 2-P-6, 141-142 (1990).

8) S. Katagiri, C.-H. Lee, and B.-H. Juang, "New discriminative training algorithms based on the generalized probabilistic descent method," Proc. IEEE-SP Workshop Neural Networks for Signal Processing, 299-308 (1991).

9) S. Amari, "A theory of adaptive pattern classifiers," IEEE Trans. Electron. Comput. 16, 299-307 (1967).

10) P.-C. Chang, S.-H. Chen, and B.-H. Juang, "Discriminative analysis of distortion sequences in speech recognition," Proc. ICASSP-91, Vol. 1, 549552 (1991).

11) T. Komori and S. Katagiri, "A new discriminative training algorithm for dynamic time warping-based speech recognition," IEICE Tech. Rep. SP91-10, 33-40 (1991).

12) P.-C. Chang and B.-H. Juang, "Discriminative template training for dynamic programming speech recognition," Proc. ICASSP-92, Vol. 1, 493-496 (1992).

13) A. Duchon, S. Katagiri, and E. McDermott, "Implementation of a prototype-based speech classifier on a fine-grained parallel computer," Proc. Autumn Meet. Acoust. Soc. Jpn. 161-162 (1991).

14) S. Katagiri and C.-H. Lee, "A new HMM/LVQ hybrid algorithm for speech recognition," IEEE 


\section{T. KOMORI and S. KATAGIRI: GPD TRAINING OF DP-BASED SPEECH RECOGNIZERS}

Proc. GLOBECOM, 608.2, 1032-1036 (1990).

15) S. Mizuta and K. Nakajima, "Optimum discriminative training for HMM with continuous mixture densities," Proc. Spring Meet. Acoust. Soc. Jpn. 1-3-12, 23-24 (1990).

16) B.-H. Juang and S. Katagiri, "A minimum error approach to speech and pattern recognition," IEEE 25-th Ann. Asilomar Conf. Signals, Systems, and Computers (1991).

17) T. Kohonen, G. Barna, and R. Chrisley, "Statistical pattern recognition with neural networks: Benchmarking studies," IEEE Proc. ICNN, Vol. 1, 61-68 (1988).

18) E. McDermott, "LVQ3 for phoneme recognition," Proc. Spring Meet. Acoust. Soc. Jpn., 151-152 (1990).

19) J. Wilpon and L. Rabiner, "A modified $K$-means clustering algorithm for use in speaker-independent isolated word recognition," AT \& T Tech. Memo., 11227-840103-1 (1984).

20) S. Amari, Information Theory II-Geometrical Thory of Information - (Kyoritsu, Tokyo, 1968), pp. 108-109.

21) A. Ando and K. Ozeki, "A clustering algorithm to minimize recognition error function," IEICE Trans. (A) J74-A, 360-367 (1991) (in Japanese).

22) K. Takeda, Y. Sagisaka, S. Katagiri, and H. Kuwabara, "A Japanese speech database for various kinds of research purposes," J. Acoust. Soc. Jpn. (J) 44, 747-754 (1988).

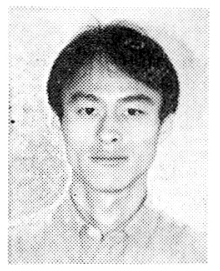

Takashi Komori was born in Toyama, Japan, on January 8, 1966 . He received the B.E. degree in mechanical engineering from the University of Tokyo, Tokyo, Japan, in 1988. In 1988, he joined INTEC Inc. Since 1990, he has been with ATR Auditory and Visual Perception Research Laboratories and a Researcher in the Hearing and Speech Perception Department. His current research interest is in speech recognition. He is a member of the Acoustical Society of Japan.

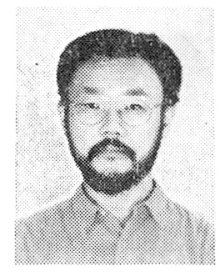

Shigeru Katagiri was born in Japan on November 3, 1953. He received the B.E. degree in electrical engineering and the M.E. and Dr. Eng. degrees in information engineering from Tohoku University, Sendai, Japan, in 1977, 1979, and 1982, respectively. From 1982 to 1986 , he worked at the Electrical Communication Laboratories, Nippon Telegraph and Telephone Public Corporation, Tokyo, Japan, where he was engaged in speech recognition research. Since 1986, he has been with the ATR Auditory and Visual Perception Research Laboratories, Kyoto, Japan, where he is currently a Supervisor in the Department of Hearing and Speech Perception. During 1989-1990, he was a Visiting Researcher with the Speech Research Department, AT \& T Bell Laboratories. He received the 22-th and 27-th Sato Paper Awards of the Acoustical Society of Japan, in 1982 and 1987, respectively. His current research interests include both theoretical studies and applications relating to discriminative training, the learning capability of artificial neural networks, and speech recognition. $\mathrm{He}$ is a member of the IEEE Signal Processing Society, the Acoustical Society of America, the Acoustical Society of Japan, the Institute of Electronics, Information and Communication Engineers, the Japanese Cognitive Science Society, and the Japanese Society for Artificial Intelligence. 